\title{
XMM-Newton observations of EF Eridani: the textbook example of low-accretion rate polars ${ }^{\star}$
}

\author{
A. D. Schwope ${ }^{1}$, A. Staude ${ }^{1}$, D. Koester ${ }^{2}$, and J. Vogel ${ }^{1}$ \\ 1 Astrophysikalisches Institut Potsdam, An der Sternwarte 16, 14482 Potsdam, Germany \\ e-mail: aschwope@aip.de \\ 2 Institut für Theoretische Physik und Astrophysik, Universität Kiel, 24098 Kiel, Germany
}

Received 13 December 2006 / Accepted 28 March 2007

\section{ABSTRACT}

\begin{abstract}
Archival X-ray observations of EF Eridani obtained in a low state revealed distinct X-ray detections at a luminosity $L_{\mathrm{X}} \simeq 2 \times$ $10^{29} \mathrm{erg} \mathrm{s}^{-1}$, three orders of magnitude below its high state value. The plasma temperature was found to be as low as $k T \lesssim 2 \mathrm{keV}$, a factor 10 below the high state. The X-ray/UV/IR spectral energy distribution suggests faint residual accretion rather than coronal emission as being responsible for the low-state X-ray emission. EFEri thus showed a clear transition from being shock-dominated in the high state to be cyclotron-dominated in the low state. From the optical/UV spectral energy distribution we re-determine the photospheric temperature of the white dwarf to $\sim 10000 \mathrm{~K}$. Contrary to earlier claims, WD model atmospheres produce sufficient UV flux to reproduce the published GALEX flux and orbital modulation.
\end{abstract}

Key words. stars: individual: EF Eri - stars: novae, cataclysmic variables - stars: magnetic fields - X-ray: binaries

\section{Introduction}

Polars are magnetic cataclysmic binaries consisting of a latetype main-sequence star and a strongly magnetic white dwarf locked in synchronous rotation. EFEri was one of the 11 polars known in the pre-ROSAT era, it was the second brightest at optical and at X-ray wavelengths after the prototypical system AM Herculis. It was studied with all major X-ray observatories (EINSTEIN, EXOSAT, GINGA, ROSAT) in the past and was always found in a high accretion state.

EINSTEIN observations revealed the presence of uncorrelated soft and hard X-ray emission and were used to observationally establish the standard picture of magnetic accretion onto white dwarfs in the high $\dot{m}$-regime dominated by a shock-heated accretion column and cooling by free-free radiation (Beuermann et al. 1987, henceforth BSP87). The absence of a pronounced soft X-ray excess made BSP87 to coin EF Eri the textbook example of AM Herculis-type systems. The shape of the X-ray light curves, in particular the presence of a soft X-ray absorption dip, was used to uncover the accretion geometry. EFEri had a main accretion pole which was continuously in view, the observer has a moderate inclination with respect to the orbital plane, so that the line of sight crosses the accretion stream on its way through the magnetosphere. This special geometry allowed detailed stream-density diagnostics with GINGA and EXOSAT (Watson et al. 1989).

The accretion geometry was intensively studied using photoand spectro-polarimetric data (e.g. Bailey et al. 1982; Cropper 1985; Piirola et al. 1987; Meggitt \& Wickramasinghe 1989; Beuermann et al. 2007). The latter three papers agree that the white dwarf's magnetic field is probably more complex than

^ Based on observations obtained with XMM-Newton, an ESA science mission with instruments and contributions directly funded by ESA Member States and NASA. that of a centered dipole. The zero point of Bailey's ephemeris (Bailey et al. 1982) centered on the IR (X-ray) absorption dip is widely used in the literature. Piirola et al. (1987) determined an updated orbital period based on a linear regression of the arrival times of linear polarisation pulses. Beuermann et al. (2007) derived a slightly revised ephemeris by including the ROSAT PSPC X-ray dip timings from July 1990. Phases in this paper refer to Bailey's phase zero and Piirola's period.

EFEri turned into a deep low state at $V \simeq 18$ in 1997 (Wheatley \& Ramsay 1998) and remains therein since then. A re-brightening was reported in VSNET on March 5, 2006 (ERI EF 20060305.724 at 14.2 unfiltered CCD based on the Henden-Sumner sequence), but the system returned to the low state shortly thereafter. While in the high state the stellar photospheres are outshone by accretion radiation, the low state offers the opportunity to investigate the stars, at least in principle. Since EFEri is the polar with the shortest orbital period, $P_{\text {orb }}=81$ min, just a few minutes above the CV minimum period, low state observations are of utmost importance to test current scenarios of $\mathrm{CV}$ evolution and to search for the cool secondary. Indeed, following the more indirect conclusion by Beuermann et al. (2000) of a substellar secondary in EF Eri from the non-detection of any spectral signature of the companion in optical spectra, Howell \& Ciardi (2001) claimed the detection of the secondary in near-infrared spectra. A more likely explanation of the observed infrared humps was given in terms of cyclotron radiation (Beuermann et al. 2000; Harrison et al. 2004).

Beuermann et al. (2000) also estimated the photospheric temperature of the white dwarf from their low-resolution optical spectra, $T_{\mathrm{WD}}=9500 \pm 500 \mathrm{~K}$, one of the coldest WDs among all CVs. This allowed to draw some conclusions on the likely evolutionary state of the object. Recently, Szkody et al. (2006) report on phase-resolved GALEX observations with the puzzling result of a distinct source of ultraviolet flux much larger than the underlying $9500 \mathrm{~K}$ white dwarf. 
Table 1. XMM-Newton observations of EF Eri. The first column lists the unique observation ID and the revolution number of the spacecraft, the last column lists the nominal and effective exposures times of the individual observations, the latter quantity after screening for high background and other instrumental defects.

\begin{tabular}{lccc}
\hline \hline OBSID/rev & Date & Instr. & $\begin{array}{c}\text { Exp Nom/Eff } \\
\text { (s) }\end{array}$ \\
\hline $0111320201 / 496$ & $2002-08-24$ & EPIC-PN & $6132 / 1859$ \\
& & OM V & 6000 \\
$0111320401 / 571$ & $2003-01-20$ & EPIC-MOS & $5160 / 5088$ \\
$0111320501 / 583$ & $2003-02-14$ & EPIC-PN & $5047 / 4559$ \\
& & EPIC-MOS & $6660 / 6575$ \\
& & OM V & 3400 \\
& & OM UVW1 & 2600 \\
\hline
\end{tabular}

Here we report on archival XMM-Newton observations of EF Eri obtained in a low accretion state. We search for remaining $\mathrm{X}$-ray emission in the low state either originating from the white dwarf or the secondary and analyse the data from the optical monitor taken through two different filters.

\section{Low-state observations with XMM-Newton}

The XMM-Newton Science Archive (XSA) contains three observations of the X-ray sky in the direction of EF Eri. They are listed with their nominal exposure times in Table 1.

We refer to individual observations by an "E" followed by the last three digits of the OBSID, i.e. E201 for the observation on 28th of April, 2002. All X-ray observations were obtained in full frame mode. The RGS did not reveal useful data due to low count rate and will not considered further. Data processing was performed with the latest version of the XMM-Newton SAS (version 7.0), a spectral analysis of the X-ray data was performed with XSPEC. Despite the relatively short exposure times, almost full phase coverage of the $P_{\text {orb }}=81$ min binary was achieved at two occasions (E401 and E501). The accumulated phase uncertainty of the period derived by Piirola et al. (1987) at the epoch of the last XMM-Newton X-ray observation, i.e. after $\sim 155000$ binary cycles, is 0.014 phase units only and thus negligible.

EPIC-MOS and EPIC-PN show a faint apparent companion to EF Eri at $\alpha(2000)=03^{\mathrm{h}} 14^{\mathrm{m}} 14^{\mathrm{s}} .0$ and $\delta(2000)=-22^{\circ} 36^{\prime} 04^{\prime \prime}$. The source has no counterpart on DSS2 images. It contributes at a level of $F_{\mathrm{X}} \simeq 1.5 \times 10^{-14} \mathrm{erg} \mathrm{cm}^{-2} \mathrm{~s}^{-1}$ in the $0.1-10 \mathrm{keV}$ band. This source could not be resolved in all previous X-ray observations with other satellites. Its faint flux was just a small contamination of all previous X-ray observations and thus irrelevant. It represents, however, a $\sim 20 \%$ contamination of the flux of EF Eri during XMM-Newton observations. We thus chose source and background regions for the extraction of light curves and spectra avoiding the region around this source.

\subsection{X-ray spectra and light curves}

The net exposure time of observation E201 was just 1859s. The source was detected with EPIC-PN at a mean count rate of $0.022 \mathrm{~s}^{-1}$. The spectrum contains no photons above $5 \mathrm{keV}$, it could be successfully fitted (reduced $\chi^{2}=0.93$ for 9 degrees of freedom) with a cooling plasma model (MEKAL in XSPEC terms) with a temperature of $k T=2.8 \pm 1.7 \mathrm{keV}$ only very little affected by interstellar absorption. In general, all X-ray spectral fits based on XMM-Newton observations are compatible with zero interstellar absorption, in accord with the low column density inferred from ROSAT and EXOSAT,

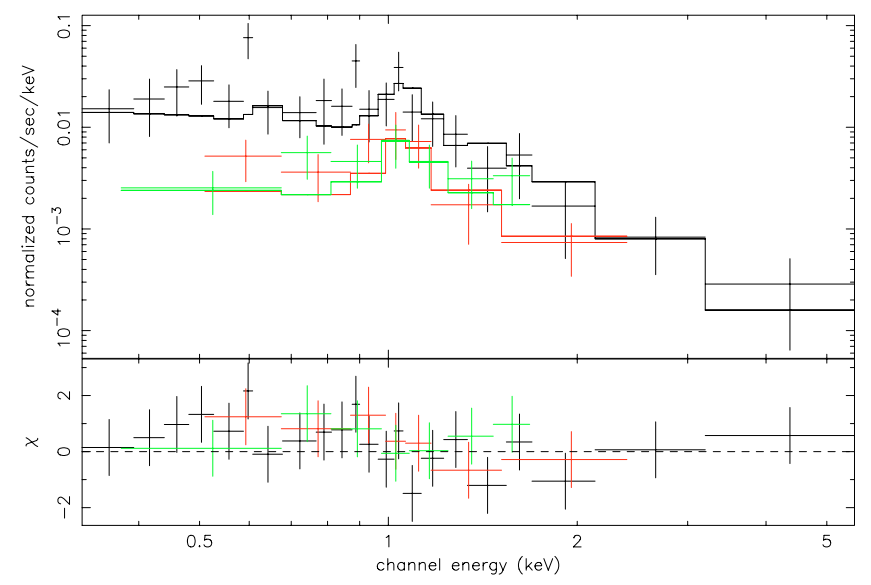

Fig. 1. Mean X-ray spectrum of EF Eri (observation E501) and best-fit thermal plasma model.

$N_{\mathrm{H}}=10^{19} \mathrm{~cm}^{2}$ (Beuermann et al. 1991; Watson et al. 1987), and from EINSTEIN, $N_{\mathrm{H}}<1 \times 10^{20} \mathrm{~cm}^{2}$ (BSP87). The integrated flux in this component was $F_{\mathrm{X}}=7 \times 10^{-14} \mathrm{erg} \mathrm{cm}^{-2} \mathrm{~s}^{-1}$ $(0.1-10 \mathrm{keV})$.

Observation E401 was performed with EPIC-MOS only and resulted in the detection of $26 / 20$ photons in 5099/5088 s with $\operatorname{MOS} 1 / 2$, respectively. The spectrum was found to be very soft again, an unconstrained fit yielded $k T=0.5 \pm 1 \mathrm{keV}$, but the spectral parameters remained highly uncertain due to the small number of photons. A fit using the same parameters as for E501, see below, yielded a flux of $F_{\mathrm{X}} \simeq 4 \times 10^{-14} \mathrm{erg} \mathrm{cm}^{-2} \mathrm{~s}^{-1}(0.1-$ $10 \mathrm{keV}$ ), slightly indicative of a lower X-ray flux at that epoch.

Observation E501 was performed with all three X-ray cameras onboard and revealed 140, 43, and 40 source photons with EPIC-PN, MOS1, and MOS2, respectively. The mean spectrum, which is a good approximation to the orbital mean spectrum also, is shown in Fig. 1. Again, it is a soft spectrum which could be fitted with just one emission component (reduced $\chi^{2}=0.81$ for 33 d.o.f.). The best-fit plasma temperature of the MEKAL model is $k T=1.7 \pm 0.2 \mathrm{keV}$, and the integrated flux $F_{\mathrm{X}}=$ $6 \times 10^{-14} \mathrm{erg} \mathrm{cm}^{-2} \mathrm{~s}^{-1}(0.1-10 \mathrm{keV})$. The O-C residuals of such a fit (see Fig. 1) give the slight impression of a systematic trend with an excess of photons between $0.5-1.0 \mathrm{keV}$. However, the parameters of any additional spectral component cannot be constrained significantly and we thus stick to a one component Xray spectral model.

We found no significant X-ray variability of the total X-ray flux between the three XMM-Newton observations. X-ray variability in E501, the longest of the three observations, was almost insignificant. A binned light curve with bin size $243 \mathrm{~s}$ (20 phase bins per orbital cycle) shows one bin with no source photon. It occurs at phase 0.7 , i.e. it cannot be associated with the high state absorption dip (if the accuracy of Piirola's period is as high as the formal uncertainties of their fit suggest). Given the small number of X-ray photons a secure claim on the existence of a dip cannot be made.

\subsection{Optical/UV observations with the OM}

The optical monitor OM was used in observations E201 and E501 with the $V$ and $U V W 1$ filters, respectively (see Table 1 for details). In E201 full phase coverage was achieved, in E501 only parts of the orbital cycle were covered with the two filters. During E201, the mean countrate in the $V$ filter (phase $0.16-1.61$ ) was $0.88(8) \mathrm{s}^{-1}$ corresponding to a 


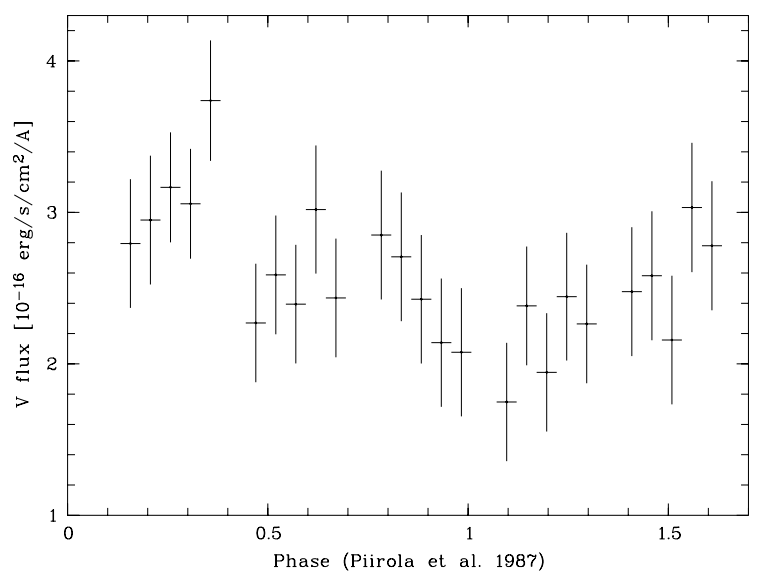

Fig. 2. OM light curve through $V$-filter from observation E201. The bin size is $243 \mathrm{~s}$ corresponding to 0.05 phase units.

mean flux of $F_{V}=2.6(2) \times 10^{-16} \mathrm{erg} \mathrm{cm}^{-2} \mathrm{~s}^{-1} \AA^{-1}$. During E501 the mean countrate through the $V$-filter (phase interval $0.90-1.55)$ was $0.87(5) \mathrm{s}^{-1}$ corresponding to $F_{V}=2.2(1) \times$ $10^{-16} \mathrm{erg} \mathrm{cm}^{-2} \mathrm{~s}^{-1} \AA^{-1}$, and through the $U V W 1$-filter (phase interval $0.67-1.17$ ) it was $1.32(4) \mathrm{s}^{-1}$ corresponding to $F_{U V W 1}=$ $6.5(2) \times 10^{-16} \mathrm{erg} \mathrm{cm}^{-2} \mathrm{~s}^{-1} \AA^{-1}$.

Some modulation of the optical flux at a level of about $50 \%$ was discovered in E201 (Fig. 2) with a minimum around phase zero. The low-state light curves by Skody et al. (2006) show a minimum at phase 0.4 . The phase difference between the two epochs by using either Bailey's period used by Szkody et al. (2006) and Piirola's period used here is negligible, 0.01 phase units. Hence, this phase shift, if a real feature of the light curve, cannot be explained by different phase conventions. However, both data sets were obtained with small telescopes and are rather noisy. Given the rather large error bars on individual light curve bins we are not further discussing possible differences between the light curves. We note, however, that the accumulated phase difference from Bailey's zero point in 1979 to the epochs of the GALEX (2004) or XMM-Newton (2003) observations is 0.18 phase units with either Bailey's or Piirola's period and thus not negligible. Formally, the period derived by Piirola et al. should be preferred due to the claimed higher accuracy, but an independent re-determination of the linear polarization ephemeris is highly desirable, should EF Eri ever return to a high accretion state.

The UVW1-filter is centered on $2910 \AA$, between the GALEX-NUV passband (Szkody et al. 2006) and the optical broad-band filters. Mean flux values as measured with the OM are shown together with other low-state photometric and spectroscopic data (Harrison et al. 2004; Szkody et al. 2006) from the infrared to the ultraviolet spectral range in Fig. 3. It shows that the different low state observations are compatible with each other.

Based on an analysis of their low-state GALEX ultraviolet and optical photometry Szkody et al. (2006) state the existence of a light-source reminiscent of a $20000 \mathrm{~K}$ hot spot. Their spot model, however, could neither explain the large-amplitude FUV variations nor the spectral energy distribution and they arrive at the conclusion, that no spot model can explain their observations.

The analysis by Szkody et al. (2006) was based on an assumed effective temperature $T_{\text {eff }}=9500 \mathrm{~K}$ (Beuermann et al. 2000) which they approximated as a Planckian function. We note, that this approximation indeed gives rise to a large ultraviolet excess. We re-address the question of the white dwarf and spot temperature making use of state-of-the-art white dwarf model atmospheres (Koester et al. 2005, and references therein). The optical spectrum alone is best described by a model with $T_{\text {eff }}=10500 \pm 1000 \mathrm{~K}$. This value is in accord with the more recent analysis by the Göttingen group (Beuermann et al. 2007) who used $T_{\text {eff }}=11000 \pm 1500 \mathrm{~K}$.

The pure white dwarf model falls short of matching the observed ultraviolet flux. We therefore fitted the SED at orbital minimum and maximum and the ultraviolet/optical light curves with a two-temperature model, a cooler one representing the white dwarf and a hotter one representing a spot. We folded our white-dwarf model spectra with the effective area curves of the two GALEX passbands ${ }^{1}$ thus converting Eddington flux to count rate. Size, temperature and location of the spot and the temperature of the white dwarf were varied until a satisfactory fit (by eye) to the optical and ultraviolet light curves and the SED were reached. We arrived at a consistent solution for $T_{\mathrm{wd}}=9750 \mathrm{~K}$ and $T_{\text {spot }}=18500 \mathrm{~K}$ with an estimated uncertainty of $1000 \mathrm{~K}$ in the white dwarf temperature. The spot temperature is subject to much larger systematic uncertainties, since e.g. our assumption of a uniform spot temperature is a very crude approximation. The model spectra shown in Fig. 3 at orbital minimum and maximum were computed for a binary inclication $i=60^{\circ}$, a spot extent of $24^{\circ}$ (half opening angle), and a spot colatitude of just $12.5^{\circ}$. A rather high inclination and a high "northern" spot latitude, the spot undergoes a partial self-eclipse only, are required from the fact that the FUV-band is almost completely dominated by the spot. Even at orbital minimum the white dwarf contributes only $\sim 10 \%$ to the total flux in that band (see the lowest model curve in Fig. 3). Our model is simple and far from being unique but it fits the data well and contradicts the conclusion by Szkody et al. (2006) that no spot model can explain both the SED and the variability.

Combining parallaxes, proper motions and absolute magnitude constraints, Thorstensen (2003) derived distance estimates for $14 \mathrm{CVs}$ with a Bayesian method, among them EF Eri. Dependent on the proper-motion, magnitude and velocity priors, he derived a short, $d=113_{-16}^{+19} \mathrm{pc}$, and a long, $d=163_{-50}^{+66} \mathrm{pc}$, distance to EF Eri. At $113 \mathrm{pc}$ the observed flux of our $9750 \mathrm{~K}$ white dwarf model results in a radius of $6.5 \times 10^{8} \mathrm{~cm}$ and implies a relatively massive white dwarf of $0.87 M_{\odot}$. At $163 \mathrm{pc}$ the implied mass is $\sim 0.55 M_{\odot}$. These numbers differ slightly from those derived in Beuermann et al. (2000), since the spot contribution was taken into account in our analysis.

\section{Results and discussion}

We have analysed archival XMM-Newton observations of EF Eri obtained in 2002 and 2003. At all three occasions the polar was detected as an X-ray source, although at a very low flux level. The spectra were compatible with emission from a lowtemperature corona-like plasma. The longest X-ray observation had almost full phase coverage, the plasma temperature was as low as $2 \mathrm{keV}$ or less. The mean orbital integrated flux in this component is about $F_{\mathrm{X}}=6 \times 10^{-14} \mathrm{erg} \mathrm{cm}^{-2} \mathrm{~s}^{-1}$. Assuming isotropic radiation and a distance of $163 \mathrm{pc}$ (Thorstensen 2003), a luminosity of $L_{\mathrm{X}} \sim 2 \times 10^{29} \mathrm{erg} \mathrm{s}^{-1}$ is derived.

The question arises if this faint $\mathrm{X}$-ray flux originates from the corona of the secondary or from the accretion region on

\footnotetext{
1 http://galexgi.gsfc.nasa.gov/tools/ Resolution_Response/index.html
} 


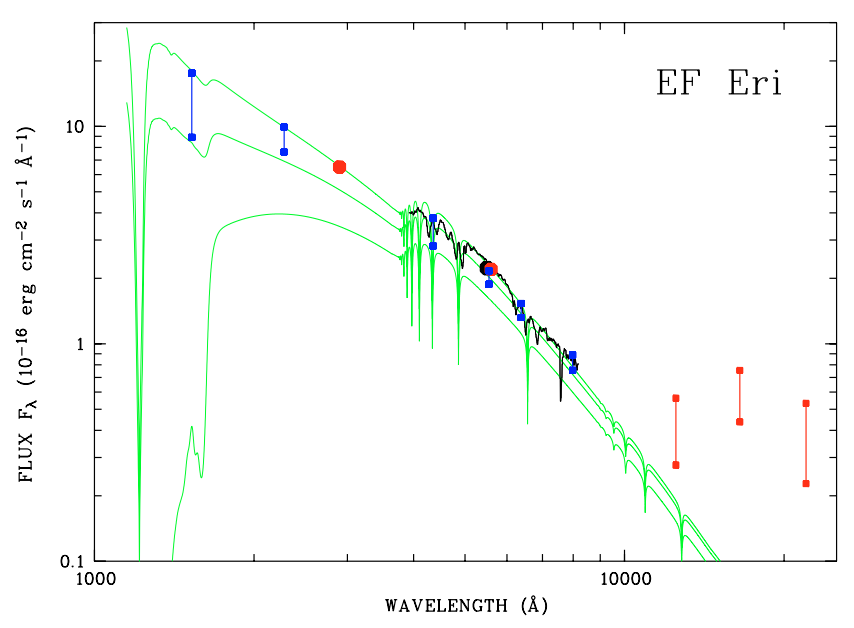

Fig. 3. Ultraviolet to infrared spectral energy distribution of EF Eri in the low state. Shown are an optical low-state spectrum and infrared $J H K$ photometry adapted from Harrison et al. (2004), GALEX-UV and optical BVRI photometry from Szkody et al. (2006, blue dots), OM $V$ band and $U V W 1$-photometry (red dots), and the result of our spectral synthesis with a two-temperature model (see text for details) Whenever possible, orbital minimum and maximum brightness are indicated and connected by lines

the white dwarf. Neither X-ray variability nor the X-ray spectrum give a clear answer. Both, a coronal plasma and the cooling plasma from low level accretion have such low temperatures as measured here. Evidence for X-ray emission from an accretion plasma can be given indirectly. Firstly, although not very much is known about X-ray emission from degenerate stars at the bottom of the main sequence, their X-ray luminosities seem to fall short by one dex with respect to the X-ray luminosiy of EF Eri (Stelzer et al. 2006). Secondly, EF Eri shows clear signs of residual accretion via the detection of infrared cyclotron harmonics (Harrison et al. 2004). It therefore appears reasonable to assign the observed X-ray emission to some remaining weak accretion. This will be our working hypothesis in the following. It remains unclear if residual accretion happens via an accretion stream or via a stellar wind, the latter being inferred in order to explain the faint X-ray emission from the small group of pre-CVs (termed also LARPs, Schwope et al. 2002; Schmidt et al. 2005; Vogel et al. 2006). Since the emission region in EF Eri is not self-eclipsing (Beuermann et al. 1987, 1991), we are lacking a distinct photometric feature to discern between the two possibilities. The pronounced soft X-ray absorption dip as a sign of stream accretion and seen in high accretion states was not secularly detected here, but due to the small number of photons its absence does not give a clear-cut answer to the question of the accretion mode.

We discuss the energy balance of the accretion process in the low state on the assumption that the observed X-ray emission is due to accretion onto the white dwarf primary. We make the further assumption that the excess emission in the infrared over the extrapolated white dwarf spectrum is solely due to cyclotron emission from the accretion plasma. The relevant radiation components are shown in Fig. 4. It shows the coronal plasma in the X-ray regime and the cyclotron component in the infrared, the latter corrected for the contribution from the underlying white dwarf. Included in the figure is the spot model at orbital maximum, represented by a white dwarf model spectrum with $T_{\text {eff }}=18500 \mathrm{~K}$.

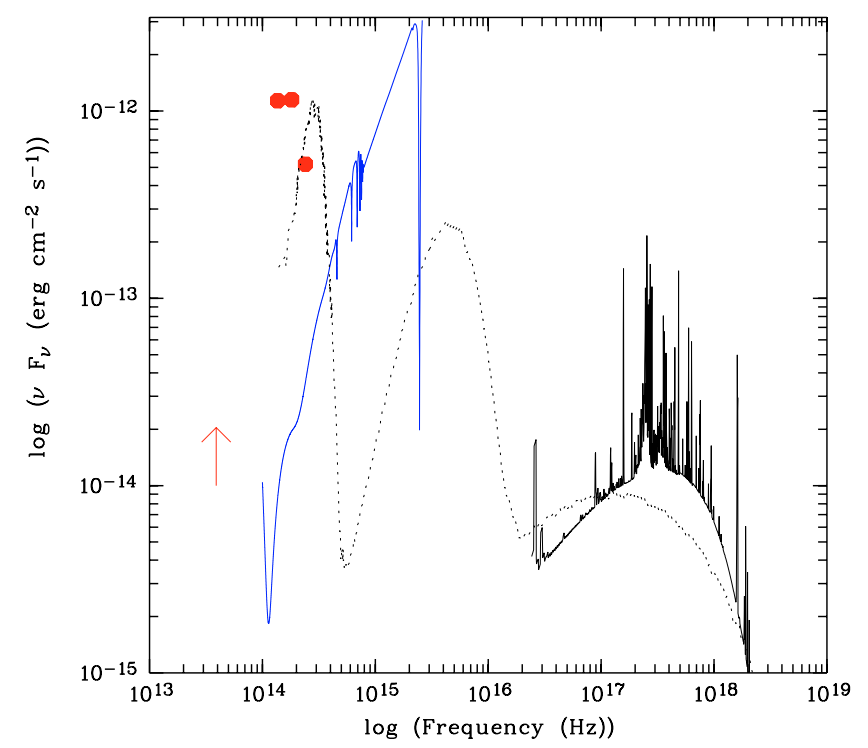

Fig. 4. Infrared to X-ray spectral energy distribution of EF Eri in the low state. Shown are radiation components which are associated with the accretion process, i.e. corrected for stellar photospheric radiation, at orbital maximum. The red arrow indicates the cyclotron fundamental for $B=14 \mathrm{MG}$.

The integrated flux in the thermal plasma X-ray component is $F_{\mathrm{X}}=6 \times 10^{-14} \mathrm{erg} \mathrm{cm}^{-2} \mathrm{~s}^{-1}$. At an assumed field strength of $13 \mathrm{MG}$ (Beuermann et al. 2007) the cyclotron fundamental is at about $8 \mu \mathrm{m}$. If we assume a Rayleigh-Jeans limited flux up to the $H$-band, where the cyclotron component peaks (Harrison et al. 2004, and Figs. 3 and 4), the integrated cyclotron flux is about $F_{\text {cyc }} \simeq 4 \times 10^{-12} \mathrm{erg} \mathrm{cm}^{-2} \mathrm{~s}^{-1}$. This value has an uncertainty of at least $50 \%$, since the cyclotron spectrum is covered only partly by observations.

There is no evidence for any component of re-processed radiation in the extreme ultraviolet/soft X-ray regime. We assume that the spot component in the ultraviolet carries the complete information on reprocessed radiation. The integrated flux in this component is of order $F_{\text {rep }} \simeq 2 \times 10^{-11} \mathrm{erg} \mathrm{cm}^{-2} \mathrm{~s}^{-1}$. This is clearly larger than the primary cyclotron radiation and a factor $\sim 300$ larger than the thermal X-ray component. The large amount of radiation in the ultraviolet in excess of the primary radiation components is suggestive of a reservoir of heat from the previous high state and does not support a picture of instantaneous reprocessing. Clearly, more UV-observations are necessary to verify this picture by determining the cooling curve of the accretion spot.

We thus derive a flux balance $F_{\mathrm{UV}} \simeq 5 \times F_{\text {cyc }}$ and $F_{\text {cyc }} \sim$ $60 F_{\mathrm{X}}$, the latter ratio being suggestive of accretion in the bombardement regime at low mass flow rates and/or high magnetic field. The field of EF Eri is one of the lowest among all polars which made the binary a rather hard X-ray source with almost balanced flux contributions in the high state (see the detailed discussion in BSP87). Since Beuermann et al. found EFEri to behave as described in the "standard" accretion scenario (Lamb \& Masters 1979), it was termed "the textbook example of AM Herculis stars" by them. This picture changed fundamentally in the low state.

Beuermann (2004) presented a sequence of model spectra for $B=14 \mathrm{MG}, \Theta=60^{\circ}$ and variable mass flow rate $\dot{m}$ (in $\mathrm{g} \mathrm{cm}^{-2} \mathrm{~s}^{-1}$ ). The values of $B$ and $\Theta$ are quite similar to those of EF Eri. We include his model for $\dot{m}=10^{-2} \mathrm{~g} \mathrm{~cm}^{-2} \mathrm{~s}^{-1}$ in Fig. 4. This model predicts the right (within an order of magnitude) flux 
ratio between cyclotron and X-ray flux. At even lower mass flow rates, the next smaller value computed by Beuermann (2004) is $\dot{m}=10^{-3} \mathrm{~g} \mathrm{~cm}^{-2} \mathrm{~s}^{-1}$, the flux ratio $F_{\text {cyc }} / F_{\mathrm{X}}$ becomes much smaller than observed. Also, the predicted size of the cyclotron emitting area would be larger than the white dwarf (for an assumed distance of $120 \mathrm{pc}$ ). We thus regard $\dot{m}=10^{-2} \mathrm{~g} \mathrm{~cm}^{-2} \mathrm{~s}^{-1}$ as the likely value for EF Eri in its low accretion state. Figure 4 shows the blackbody approximation as non-appropriate for the reprocessed component. This was noted already by Beuermann (2004), the low state observations of EF Eri prove this observationally.

At $\dot{m}=10^{-2} \mathrm{~g} \mathrm{~cm}^{-2} \mathrm{~s}^{-1}$ and $B=13 \mathrm{MG}$ the maximum predicted electron temperate is about $7 \mathrm{keV}$ (Fischer \& Beuermann 2001). Our measured temperature $k T \simeq 1.7 \mathrm{keV}$ (E501) indicates that the bulk of X-ray emission originates from denser layers at lower temperatures, as expected.

A comparison between high and low state fluxes of the main radiation components is instructive. For the mean fluxes in the high state, BSP87 derive $F_{\text {cyc }}=4.8 \times 10^{-11} \mathrm{erg} \mathrm{cm}^{-2} \mathrm{~s}^{-1}$, $F_{\text {brems }}=1.5 \times 10^{-10} \mathrm{erg} \mathrm{cm}^{-2} \mathrm{~s}^{-1}$, and $F_{\text {bb }}=5.5 \times$ $10^{-10} \mathrm{erg} \mathrm{cm}^{-2} \mathrm{~s}^{-1}$, respectively. Hence, when switching from the high to the low state, the cyclotron flux is reduced by a factor $\sim 10$, and the flux in the thermal plasma component by a factor 2500. These numbers illustrate the variable occupation of the different channels of energy release, when switching from a high-accretion rate, shock-dominated flow to the low-rate, cyclotron-dominated bombarded atmosphere. A direct comparison between the high- and low-state fluxes in the re-processed component seems not to be possible, since a counterpart to the high-state blackbody is missing in the low state. The low-state ultraviolet flux does not seem to be due to instantaneous reprocessing but still to be fed from high-state accretion heating.

The bolometric flux in the low state is $F_{\text {acc }} \simeq F_{\text {cyc }} \simeq$ $4 \times 10^{-12} \mathrm{erg} \mathrm{cm}^{-2} \mathrm{~s}^{-1}$, the luminosity is $L_{\mathrm{acc}} \simeq 2 \pi d^{2} F_{\mathrm{acc}} \simeq$ $2.4 \times 10^{30} d_{100}^{2} \mathrm{erg} \mathrm{s}^{-1}$, a factor 100-300 lower than the high state accretion luminosity derived by BSP87.

We may also compare the cyclotron emitting areas in the high and low states. BSP87 derive $A_{\text {cyc }}=(3.2-12.2) \times$ $10^{15} d_{100}^{2} \mathrm{~cm}^{2}$ while scaling of the model shown in Fig. 4 implies $A_{\text {cyc }} \simeq 30 \times 10^{15} d_{100}^{2} \mathrm{~cm}^{2}$. Again, care has to be taken by taking the latter number too literally since we have just scaled a preexisting model, but they imply that the cyclotron emitting area has not shrunk by orders of magnitude.

A final comment may be made on EFEri as a polar and its relation to the class of (likely misleadingly termed) LARPs (Low-Accretion Rate Polars; Schwope et al. 2002). The latter are close white dwarf/M dwarf pairs with pronounced cyclotron harmonics which led to their discovery in the HQS and the SDSS spectroscopic surveys. They are likely detached binaries acrreting from the stellar wind of the secondary (Schmidt et al. 2005;
Vogel et al. 2007). EF Eri is in a deep low state now for about 10 years. It is faint, its optical spectrum shows just the white dwarf and no secondary, variability in the optical is small, $\Delta V<$ $0.3^{m}$, its X-ray flux is faint and below all flux limits of surveys with sufficiently large survey area. Hence, all the classical discovery channels of CVs would not have led to the identification of EF Eri as a rather nearby cataclysmic variable in its extended low state. It would have been classified just as an isolated magnetic white dwarf in a spectroscopic survey.

But it shows an infrared excess, which could be (erroneously) interpreted as originating from a secondary and therefore would hint to the binary nature of this source. Actually it still shows a high degree of variability, although most pronounced in the ultraviolet. And finally, it still emits X-rays, but at the given flux the X-ray sky is dominated by AGNs. Hence, it still shows many hallmarks of the polars. and in that respect we may coin EFEri as the textbook example of the low-accretion rate polars. We are not going to speculate about a population of missing CVs in similar extended low states. But the case of EFEri as a secure low-accretion rate polar underlines the importance of a multi-wavelength approach to find more of these intriguing sources.

Acknowledgements. We thank our referee, Klaus Beuermann, for helpful comments which improved the manuscript. We thank V. Hambaryan and G. Lamer for help with the data reduction. This work was supported in part by the Deutsches Zentrum für Luft- und Raumfahrt (DLR) GmbH under contract No. FKZ 50 OR 0404 and by the Deutsche Forschungsgemeinschaft DFG under contract No. Schw536/20-1.

\section{References}

Bailey, J., Hough, J. H., Axon, D. J., et al. 1982, MNRAS, 199, 801

Beuermann, K. 2004, ASPC, 315, 142

Beuermann, K., Stella L., \& Patterson, J. 1987, ApJ, 316, 360 (BSP87)

Beuermann, K., Wheatley, P., Ramsay, G., Euchner, F., \& Gänsicke, B. T. 2000, A\&A, 354, L49

Beuermann, K., Euchner, F., Reinsch, K., Jordan, S., \& Gänsicke, B. T. 2007, A\&A, 463, 647

Cropper, M. 1985, MNRAS, 212, 709

Harrison, T. E., Howell, S. B., Szkody, P., et al. 2004, ApJ, 614, 947

Koester, D., Napiwotzki, R., Voss, B., Homeier, D., \& Reimers, D. 2005, A\&A, 439,317

Meggitt, S. M. A., \& Wickramasinghe, D. T. 1989, MNRAS, 236, 31

Nauenberg, M. 1972, ApJ, 175, 417

Piirola, V., Reiz, A., \& Coyne, G. V., S. J. 1987, A\&A, 186, 120

Schmidt, G. D., Szkody, P., Vanlandingham, K. M., et al. 2005, ApJ, 630, 1037

Schwope, A., Brunner, H., Hambaryan, V., et al. 2002, ASPC, 261, 102

Stelzer, B., Micela, G., Flaccomio, E., Neuhäuser, R., \& Jayawardhana, R. 2006, A\&A, 448, 293

Szkody, P., Harrison, T. E., Plotkin, R. M., et al. 2006, ApJ, 646, L147

Thorstensen, J. R. 2003, AJ, 126, 3017

Vogel, J., Schwope, A. D., \& Gänsicke, B. T. 2007, A\&A, 464, 647

Watson, M. G., King, A. R., Jones, M. H., \& Motch, C. 1989, MNRAS, 237, 299

Wheatley, P. J., \& Ramsay, G. 1998, ASPC, 137, 446 